This item was submitted to Loughborough's Research Repository by the author.

Items in Figshare are protected by copyright, with all rights reserved, unless otherwise indicated.

Soft tissue motion during impacts: their potential contributions to energy dissipation

PLEASE CITE THE PUBLISHED VERSION

http://journals.humankinetics.com/JAB

PUBLISHER

(c) Human Kinetics, Inc.

VERSION

AM (Accepted Manuscript)

LICENCE

CC BY-NC-ND 4.0

REPOSITORY RECORD

Pain, Matthew T.G., and John H. Challis. 2019. "Soft Tissue Motion During Impacts: Their Potential Contributions to Energy Dissipation”. figshare. https://hdl.handle.net/2134/6531. 
This item was submitted to Loughborough's Institutional Repository (https://dspace.lboro.ac.uk/) by the author and is made available under the following Creative Commons Licence conditions.

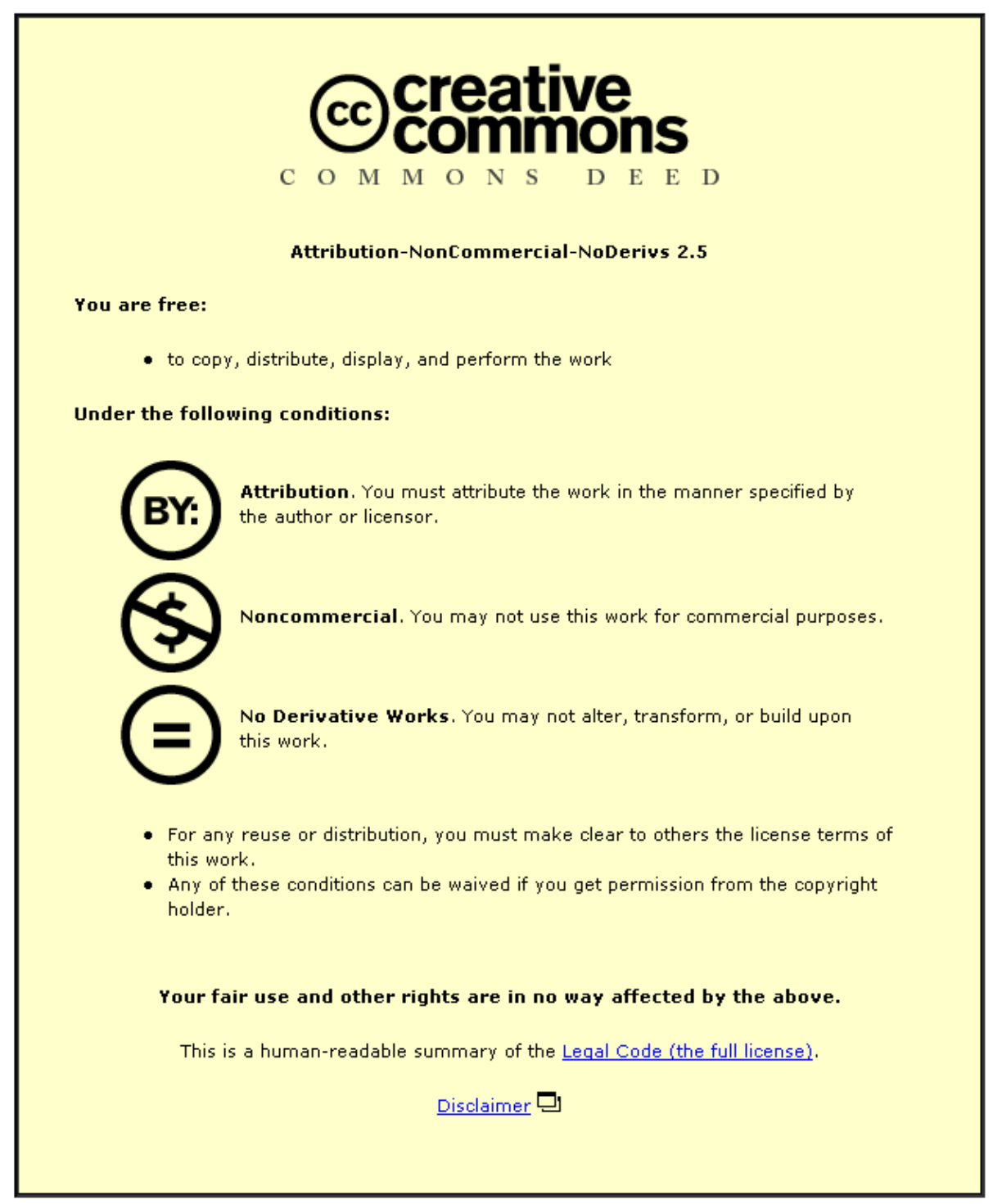

For the full text of this licence, please go to: http://creativecommons.org/licenses/by-nc-nd/2.5/ 


\title{
Soft tissue motion during impacts: Their potential contributions to energy dissipation
}

\author{
Matthew T.G. Pain ${ }^{1}$ and John H. Challis ${ }^{2}$ \\ ${ }^{1}$ School of Sport and Exercise Sciences, Loughborough University, United Kingdom \\ ${ }^{2}$ Biomechanics Laboratory, The Pennsylvania State University, PA 16802-3408, USA
}

\begin{abstract}
The aims of this study were to quantify intra-segmental motion using an array of 28 surface mounted markers to examine frequency and amplitude measurements of the intra-segmental motion to calculate forces and energy transfer; and to show that the underlying muscles are a major contributor to the skin marker motion. One subject performed 27 trials under three conditions in which his forearm was struck against a solid object fixed to a force plate while the locations of the markers were recorded at $240 \mathrm{~Hz}$. For impacts with equal peak forces the muscle tension significantly affected the amount of intra-segmental motion. Tensing the arm reduced the intra-segmental motion by $50 \%$. The quadrilateral sectors defined by the markers changed in area by $11 \%$ with approximately equal motion in the vertical and horizontal direction. The maximum linear marker motion was $1.7 \mathrm{~cm}$. The intra-segmental motion had distinct frequency components around 14 and $20 \mathrm{~Hz}$. Soft tissue deformation could account for $70 \%$ of the energy lost from the forearm during these impacts. The study has demonstrated the important role that intra-segment soft tissue motion can have on the kinetics of an impact.
\end{abstract}

KEYWORDS: marker arrays, intra-segmental motion, impacts

\section{INTRODUCTION}

Human movement is often punctuated by a series of impacts, for example heel strike during walking or running. During impacts the dissipation of the energy associated with the impact can be important. The human heel pad has been identified as an important site for this energy dissipation (e.g. Aerts, Ker, Ilsley \& Alexander, 1995). In an analysis of landing from a drop Zatsiorsky \& Prilutsky (1987) estimated that up to $75 \%$ of the mechanical energy of the body could be dissipated passively. The heel pad could not be responsible for all of this energy dissipation so there must be additional sites. One potential source is the motion of the soft tissue of the body segments. During impacts motion of the soft tissue independent of the skeleton is clearly seen, this may be a significant site of energy dissipation.

Studies comparing the motion of skin mounted markers with those directly attached to the bone of the same segment via cortical pins during impacts show relative motions of up to 7 cm (Lafortune, Lambert, \& Lake, 1992). Studies looking at slow movements have also shown intra-segmental motion (Karlsson \& Lundberg, 1994; Fuller, Liu, Murphy, \& Mann, 1997) but it is most pronounced as a consequence of impacting types of activities (Reinschmidt, van den Bogert, Nigg, Lundberg, \& Murphy, 1997). This relative motion between bone and skin mounted markers is often referred to as the skin movement artifact. Cappozzo, Catani, Leardini, Benedetti, \& Della Croce (1996) stated that skin movement artifact can produce the most significant errors in human movement analysis as the location of the skeleton and hence joints could not be accurately located.

Examining the artifact just to remove it seems to be ignoring the fact that to cause intrasegmental motion forces must be acting differentially within the segment and altering the kinetics from that of a rigid body. If the marker motion is totally random noise then it is probably best to attempt to remove it from the analysis all together. However if the skin movement artifact is not the result of purely random processes then it should have discernible 
structure. The point cluster method (Andriacchi, Alexander, Toney, Dyrby, \& Sum, 1997) minimises the influence of intra-segmental array marker motion using optimisation techniques in an attempt to remove this movement artifact to improve accuracy of measurement of skeletal motion. Knowledge of the impulse response of the soft tissue motion may allow a faster and more accurate solution using this type of technique.

Removing soft tissue motion that is structured removes a real set of forces from kinetic analyses. This would be especially true if the surface marker motion is dependant on the underlying soft tissue motion that constitutes the majority of limb mass. A number of studies using models have examined intra-segmental soft tissue motion, wobbling mass models, and have indicated that these wobbling masses can cause markedly different force responses compared with rigid body models (e.g. Gruber, Ruder, Denoth, \& Schneider, 1998; Liu \& Nigg, 2000; Pain \& Challis 2001a).

During an impact the deformation occurs at one end of the limb and an unbalanced force is produced. As the limb materials have visco-elastic properties the materials will attempt to return to equilibrium when the force is removed. This will result in a transient deformation, seen as a wave. The general form of the wave equation is given by equation 1 ,

$$
\frac{\partial^{2} \psi}{\partial t^{2}}=V^{2} \cdot \nabla^{2} \psi
$$

Where

$\psi$ is the dilation (change in volume / original volume) or rotation about the axis parallel to the line of propagation

$V$ - velocity of the wave

$\nabla \quad$ - Del operator - $\quad i \frac{\partial}{\partial x}+j \frac{\partial}{\partial y}+k \frac{\partial}{\partial z}$

where $\mathrm{i}, \mathrm{j}$, and $\mathrm{k}$ are unit direction vectors

The velocity $(V)$ is related to the mechanical properties of the medium within which the wave is travelling by equation 2 .

$$
V=\sqrt{\frac{C}{\rho}}
$$

$\mathrm{C}$ is the constant relating stress and strain usually of the form of Youngs modulus or a non-linear variant

$\rho$ is the density of the medium within which the wave is propagating

In comparison with the skin the muscle-tendon complex has high stiffness (Fung, 1981). Therefore the deformation wave due to an impulsive loading will not be due to skin motion as much as it is due to the motion of the underlying muscle because of the low stiffness of the skin at low strains not propagating forces well.

Waves of some description will pass along the tissues of the limb. Depending on the nature of the applied force, the boundary conditions and mechanical properties of the tissues it should be possible to measure some of the wave motion with arrays of markers on the surface of a limb. Pure compression waves are unlikely due to the majority of soft tissue not being rigid in compression. Current motion analysis techniques probably preclude identification of the type of wave produced, but estimates of energy transfer and forces can be calculated if the frequencies and amplitudes of the wave motion can be determined. The wave motion travelling along the limb will be referred to as a tissue wave. 
An estimate of the energy carried by the tissue wave can be obtained by using the equation for the energy density of a non-dispersive wave propagating in one direction,

$$
E d=\frac{1}{2} \rho \cdot A^{2} \cdot \omega^{2}
$$

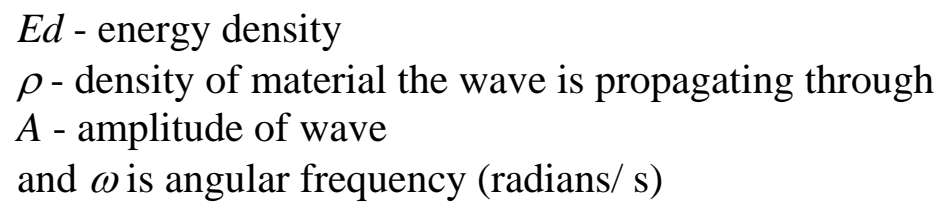

Precise calculations can be problematic if the waves propagating appear to be dispersive. A dispersive wave is one where the velocity of the wave is dependent on the frequency of the wave (Graff, 1991). For mechanical waves in an elastic uniform medium dispersion does not normally occur. Non-uniform media and anisotropic media, as are present in the soft tissue of the human body are normally dispersive (Maurel, Thalmann, \& Magnenat-Thalmann, 1998). The energy transmitted by a wave as calculated by equation 3 , is only an estimate as the assumptions have been made that: all measurements are accurate; the tissue wave is nondispersive; and that it is a uni-directional compression wave.

The purpose of the study is to present how frequency and amplitude measurements of intrasegmental motion are highly dependant on the muscle mass and how these measures can be used to calculate the force and energy transfer within a body segment. Experimental work is presented to demonstrate the amount of energy that can be dissipated using such a mechanism.

\section{METHODS}

The task to be undertaken required a skilled subject to perform a repetitive striking task, therefore one male subject was recruited who had 19 years training in martial arts (age 27, height $1.75 \mathrm{~m}$, weight $85 \mathrm{~kg}$, body fat $10 \%$ ). The subject provided informed consent, and all procedures were approved by the institutional review board. The subject had an array of 28 circular reflective markers placed on the anterior aspect of his right forearm. The markers were each $3 \mathrm{~mm}$ in radius, and had a mass of 0.0057 grams which was sufficiently low so that their inertia did not produce motion independent of the skin to which they were attached. The array of markers was 4 markers across and 7 markers down the forearm from $1 \mathrm{~cm}$ below the axis of rotation of the elbow to $8 \mathrm{~cm}$ above the wrist. This gave 18 quadrilateral sectors defined by a marker at each vertex. The coordinates of the reflective marker were recorded in two dimensions by a Qualysis Pro-Reflex system at $240 \mathrm{~Hz}$ for three seconds per trial.

The subject performed 27 strikes onto the top of a solid wooden platform that had been fixed to a Bertec force plate (model 4060s) recording at $1200 \mathrm{~Hz}$, synchronized electronically with the Qualysis Pro-Reflex system. Each strike was a downward straight strike (atoshi teisho uchi) with the long axis of the forearm remaining vertical and perpendicular to the camera from just before impact until the arm was stationary against the wooden platform (Figure 1). The subject was instructed to produce a relatively light impact and to try to produce the same strike each time. Contact was made with the proximal end of the palm, which was extended to lie directly over the radius and ulna. During the first set of nine strikes the subject was asked to keep his forearm musculature as relaxed as possible, the loose condition. For the second set of nine strikes the subject was asked to 'stiffen' these muscles and produce a similar impact velocity, the stiff condition. For the third set of nine strikes the subject was asked to provide an impact with similar impact forces to the first set of strikes but with the 
forearm musculature as stiff as possible, the rigid condition. The rigid condition necessitated a lower impact velocity than the other two conditions

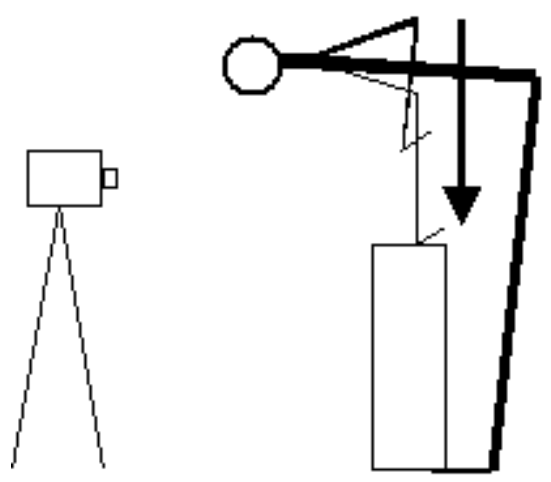

Figure 1. Subject performing the strike. The arrow indicates direction of motion.

The inertial parameters of the forearm were calculated using a sonic digitizer as reported in Pain \& Challis (2001b) for the arm in a loose state. This technique allows body segment inertial parameters to be calculated by obtaining random surface data points of the body under investigation, interpolating them into a regular grid to give the dimensions of the object and then using Greens theorem which relates surface to volume integrals.

The data were analyzed for two purposes. The first involved a statistical analyses of the peak vertical ground reaction force and deformation of the marker array for each set of nine strikes. The second set of analyses were performed only on the nine strikes in the loose condition to examine the characteristics of the deformation of the array. Array deformation was characterized by calculating the changes of area of the quadrilaterals defined by markers at their vertices.

The majority of the analyses of the markers was carried out only on the data from just prior to impact until the arm was stationary to remove the large amplitude low frequency components associated with pre-impact motion. Pre-impact data were kept separate and used to calculate the velocity of the arm at impact. Kinematic variation was kept to a minimum between the loose and the stiff condition by having the initial orientation and orientation of the arm at impact the same between trials and by endeavoring to keep the impact velocity constant between conditions. The data of the motion of the forearm and hand prior to impact were used to compute the kinetic energy of the forearm at impact. To compare the changes in marker motion the loose condition and the rigid condition were used as they had similar impact forces. Using trials with higher impact forces would be expected to increase intrasegmental motion due to the greater stress on the tissues. The maximum change in area for each sector was averaged across all trials, for both the loose condition trials and the rigid condition trials. Further analysis was only carried out on the loose condition data, as the interest is in observing the soft tissue deformation, which was significantly greater in these trials.

The raw data for the sector areas were examined and low pass filtered at $25 \mathrm{~Hz}$ before further analysis. The majority of sector data had a distinct drop in the power spectrum between 20$25 \mathrm{~Hz}$ and approximately 90\% of the power was below $25 \mathrm{~Hz}$. Frequency analysis was performed in Matlab and spectral powers were determined using a windowed FFT method. Next the delay between the proximal and distal markers recording the impact was found to give an estimate of the speed of propagation of the tissue wave caused by the impact. The delay in the peak amplitude of the wave to travel from the proximal to the distal markers was 
also calculated from the delay in peak amplitude between the most proximal marker and the more distal marker. The mean peak amplitude of the wave was measured for each marker and each sector for each trial. Finally the energy carried by the wave was calculated using an equation for energy density and the inertial parameters of the forearm (equation 3).

The peak impact force and the time from initial contact to peak impact force were compared between conditions using an ANOVA, and where appropriate with a post hoc Tukey (HSD) test. The maximum change in area, averaged over the nine trials in each condition, for each sector was compared between the loose condition and the rigid condition using a paired t-test. The significance level was set at 0.01 for both tests.

\section{RESULTS}

The mean and standard deviation for the peak vertical impact forces were $712 \pm 90 \mathrm{~N}, 1023 \pm$ $112 \mathrm{~N}, 806 \pm 130 \mathrm{~N}$ for the loose, the stiff, and the rigid condition respectively. There was no significant difference ( $>0.01)$ between the peak impact force between the loose condition and the rigid condition. There was a significant difference $(p<0.01)$ between the loose condition and the stiff condition. The time from initial impact to these peak forces were $0.01825 \pm 0.003 \mathrm{~s}, 0.011 \pm 0.003 \mathrm{~s}$, and $0.012 \pm 0.0025 \mathrm{~s}$ for the loose, the stiff, and the rigid conditions respectively. For the times to peak force there was a significant difference $(\mathrm{p}<$ 0.01 ) between the loose condition and both the stiff and rigid condition. There was no significant difference $(\mathrm{p}<0.01)$ between the loose and rigid conditions for the pre-impact velocity. Across all sectors the loose condition had twice the change in area that the rigid condition had. Fourteen of the 18 sectors showed significantly more change in area across the nine loose trials when compared with the rigid condition.

Analyzing each marker for each trial in the loose condition showed that similar results were seen across markers for motion in both the vertical and horizontal directions. Immediately post impact the marker continues to move downward for $0.0125 \mathrm{~s}$, due to the soft tissue on the palm compressing and the inertia of the soft tissue. The marker then moves up past its final stationary position before two further small oscillations can be seen of approximately equal period before it comes to rest (Figure 2).

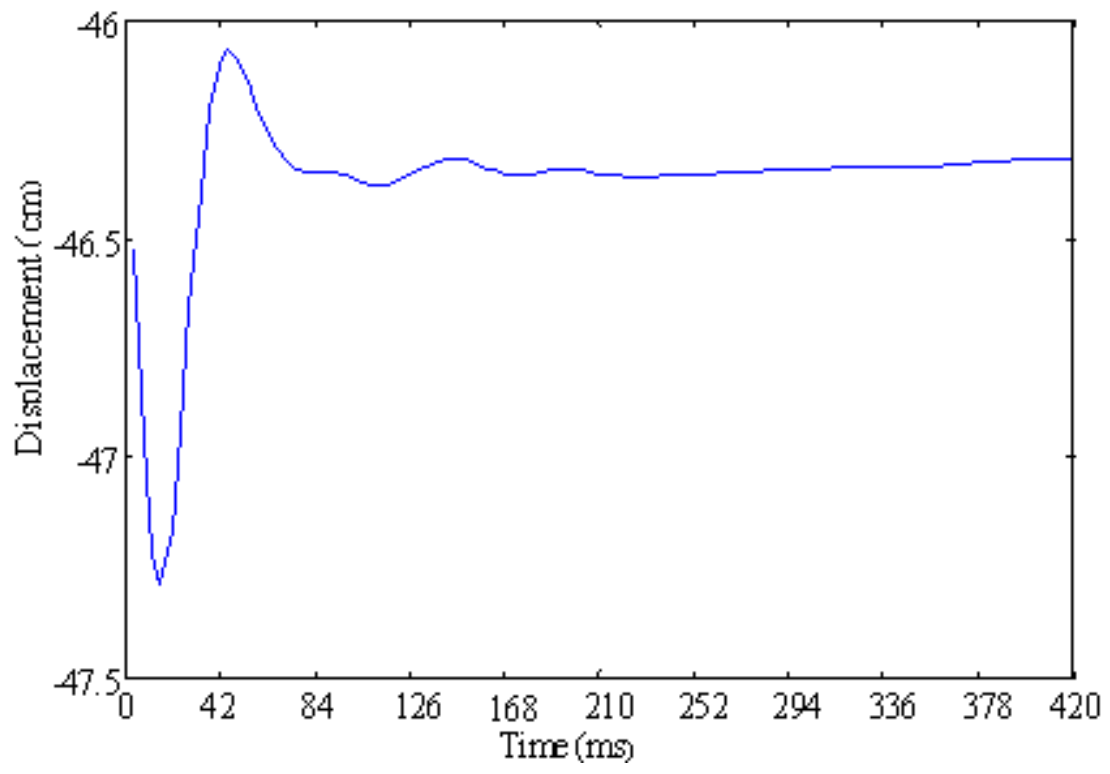

Figure 2. Displacement of a typical marker in the vertical direction after impact at $\mathrm{t}=0$. 
Analyzing each sector for each trial also showed similar results across sectors (Figure 3). A definite damped oscillation in the change of area can be seen immediately following impact. This can be seen running longitudinally along the arm (Figure 4). The mean area of each sector was $4.5 \mathrm{~cm}^{2}$ in the resting condition and the mean variation in area was $11 \%$ (Table 1 ).
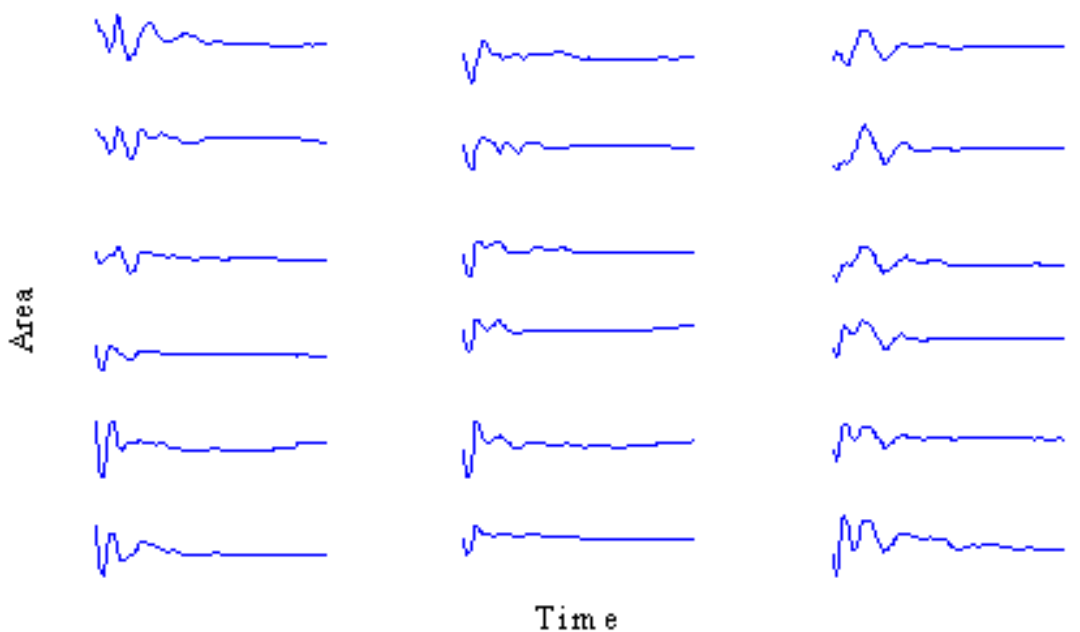

Figure 3. Change in area of each sector with time for a trial. The change in area is up to $1 \mathrm{~cm}^{2}$ for a resting area of $4 \mathrm{~cm}^{2}$ to $7 \mathrm{~cm}^{2}$ and the time axis for each sub-plot lasts 0.5 seconds. Top right sector corresponds to the proximal ulnar portion of the forearm while the bottom left corresponds to the distal radial portion.

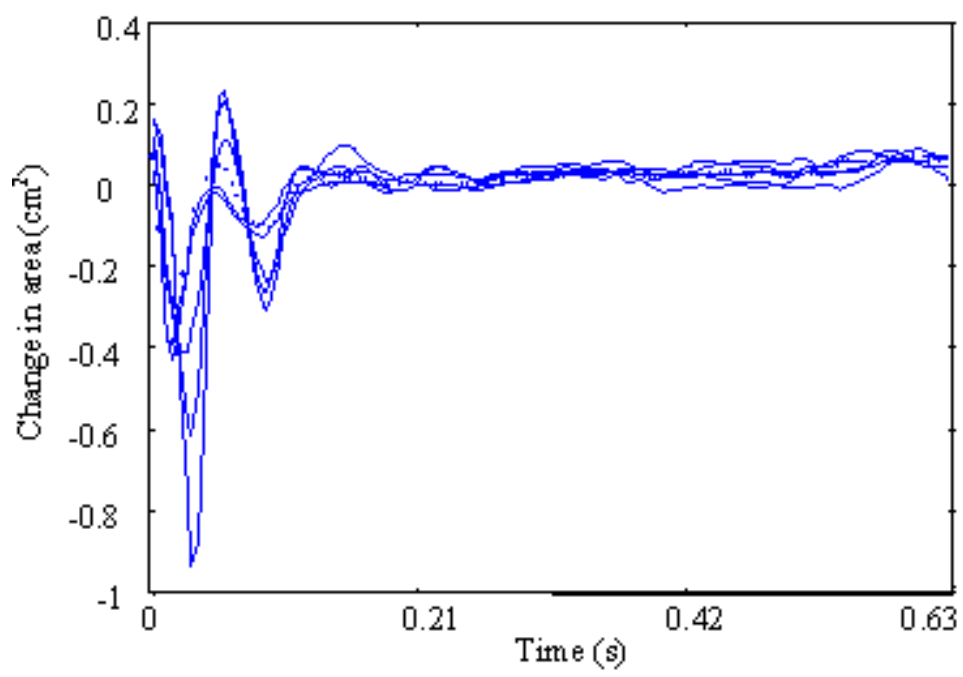

Figure 4. Changes in area for one column of areas. Each line refers to one sector in a column. This shows each sector expands and contracts with approximately the same phase.

The frequency content of the maker displacement data predominately showed a double peak for the vertical coordinate data with a typical low frequency peak around $3 \mathrm{~Hz}$ and a second peak between 11 and $20 \mathrm{~Hz}$ (Figure 5A). Occasional other higher frequency peaks were seen at 9-11 $\mathrm{Hz}$ and at $22 \mathrm{~Hz}$ (Table 1). The peak frequency horizontally was usually $1-2 \mathrm{~Hz}$ greater than that of the second peak in the vertical direction. The frequency content of the sector data tended to have a single or double peak of approximately equal magnitude as well as a low frequency peak. These peaks were centered around $12 \mathrm{~Hz}, 15 \mathrm{~Hz}$ or $20 \mathrm{~Hz}$ (Figure 5B). 
Table 1. The mean ( \pm standard deviation) across sectors for the change in displacement of the markers in the vertical direction, average of 28 markers, and the change in area of the sectors, average of 18 sectors, as well as the median peak frequency of the second peak in the frequency spectrum of the areas and the markers, for the loose condition.

\begin{tabular}{|c|c|c|c|c|}
\hline Trial & $\begin{array}{c}\text { Mean } \Delta \text { position } \\
(\mathrm{cm})\end{array}$ & $\begin{array}{c}\text { Mean } \Delta \text { Area } \\
\left(\mathrm{cm}^{2}\right)\end{array}$ & $\begin{array}{c}\text { Median of } 2^{\text {nd }} \text { peak } \\
\text { for markers }(\mathrm{Hz})\end{array}$ & $\begin{array}{c}\text { Median of } 2^{\text {nd }} \text { peak for } \\
\text { sectors }(\mathrm{Hz})\end{array}$ \\
\hline 1 & $2.1 \pm 0.4$ & $0.61 \pm 0.05$ & 14 & 14 \\
\hline 2 & $1.7 \pm 0.3$ & $0.50 \pm 0.05$ & 12 & 12.5 \\
\hline 3 & $1.8 \pm 0.3$ & $0.49 \pm 0.06$ & 12 & 13 \\
\hline 4 & $1.5 \pm 0.2$ & $0.55 \pm 0.07$ & 11 & 13 \\
\hline 5 & $1.3 \pm 0.2$ & $0.41 \pm 0.04$ & 14 & 15 \\
\hline 6 & $1.2 \pm 0.2$ & $0.56 \pm 0.07$ & 13 & 14 \\
\hline 7 & $1.6 \pm 0.1$ & $0.42 \pm 0.05$ & 19 & 15 \\
\hline 8 & $1.9 \pm 0.2$ & $0.48 \pm 0.05$ & 14 & $14.1 \pm 2.1$ \\
\hline 9 & $1.9 \pm 0.2$ & $0.41 \pm 0.05$ & 12 & 13 \\
\hline Mean & $1.7 \pm 0.3$ & $0.49 \pm 0.06$ & $13.4 \pm 2.2$ & 14 \\
\hline
\end{tabular}
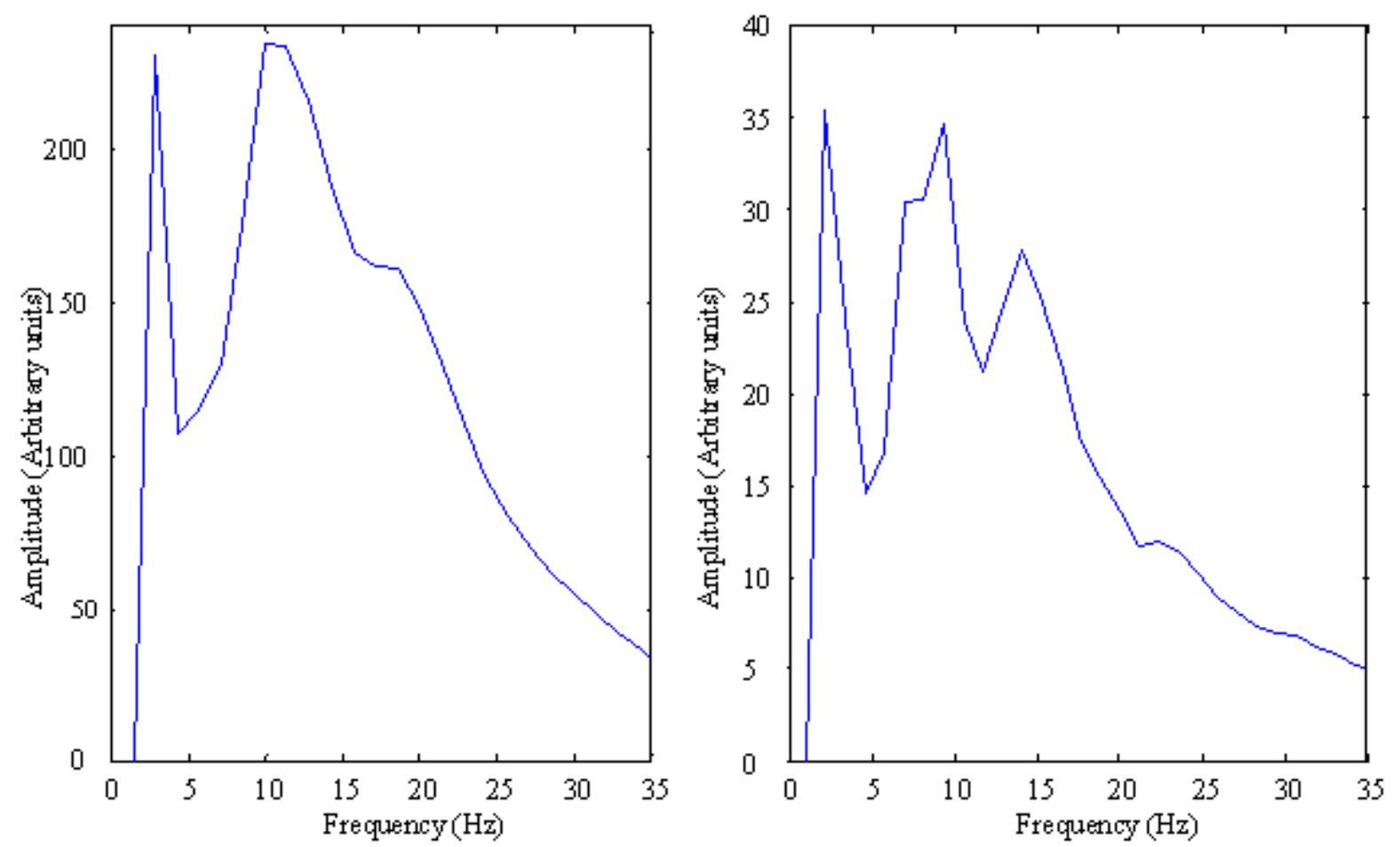

Figure 5. Frequency content summed across all markers for one trial, A, and across all sectors (areas) for another trial, B 
The delay between the proximal and distal markers responding to the impact was found to be $0.0042 \mathrm{~s}$. Propagation of the impact by a mechanical wave gave a velocity of $37 \mathrm{~m} / \mathrm{s}$.

The mean cross sectional area of the soft tissue of the forearm was estimated at $43 \mathrm{~cm}^{2}$ using the inertial parameters determined from the sonic digitizer and data on tissue distribution from Clarys \& Marfell-Jones (1986) and Clarys, Martin, \& Drinkwater (1984). Density of the soft tissue was assumed to be $1020 \mathrm{~kg} / \mathrm{m}^{3}$ (Clarys \& Marfell-Jones, 1986; Clarys, Martin, \& Drinkwater, 1984). The length of the forearm was $0.26 \mathrm{~m}$. Mass of the soft tissue was calculated to be $1.14 \mathrm{~kg}$. Using the mean amplitude, half the value in table 1 , and the frequency for the markers presented in table 1 with equation 3 gives an energy density of 261 $\mathrm{J} / \mathrm{m}^{2}$. Therefore energy carried by the wave through the soft tissue was $1.1 \mathrm{~J}$. The mean kinetic energy across trials of the forearm at impact was obtained from the mass of forearm, $1.38 \mathrm{~kg}$, and the mean velocity at impact, $1.5 \mathrm{~m} / \mathrm{s}$, and was $1.6 \mathrm{~J}$. There was no rotation of the forearm.

\section{DISCUSSION}

By altering the tension of the muscles in the forearm the kinetics of the impact were altered when the kinematics, as defined by velocity and orientation at impact of the body segment, were left unaltered. Increasing the 'stiffness' of the muscles increased the peak impact force and decreased time to peak force. Stiffening the system decreased the time to peak impact more so than increasing impact velocity. This would indicate that it was not purely the palm heel pad deformation that was governing the force interaction over time. The soft tissue of the forearm was decelerated quicker when it was stiffer, giving a greater effective mass in the impact.

Comparing the array deformation between the loose and rigid conditions the effect of altering the tension in the underlying muscle on the skin motion was quantitatively established for a limited range of muscle tensions and impact forces. Given that stiffer muscles would undergo less deformation for an equivalent stress and with the stiffness of the skin unchanged between conditions, the decrease in array deformation can be attributed to the decrease in muscle deformation. In a high impact activity it is likely that nearly all skin marker motion can be attributed to the underlying muscle deformation.

The change in vertical position of the markers after impact of up to $2 \mathrm{~cm}$ is less than skin movement artifacts reported by Lafortune, Lambert, \& Lake (1992) and Reinschmidt (1996) for the leg during human gait. Both of those studies used cortical pins so marker motion could be compared to bone motion. In the present study bone motion was not directly measured, so it could be argued that the marker motion is entirely due to whole limb motion rather than intra-segmental soft tissue motion. Although possible, examining the data did not indicate that this was likely to be the major contributor as analysis of marker movement commenced once contact had been made, and once in contact the hand remained in contact. Part of the measured marker motion after impact is likely to be due to the palm heel deformation allowing whole limb motion. Examining the maximum static depth of compression for the impact area of the palm heel for the subject gave a compression depth of $3.5 \mathrm{~mm}$.

The measurement of sector deformation was independent of whole limb motion and showed deformations of $11 \%$ change in area, defined by maximum area minus minimum area divided by the mean area, for each sector clearly indicating soft tissue motion as a consequence of the impact. Without being able to directly associate the change in area with changes in the underlying volume, dilation, rotation, shearing of muscle, calculating the forces involved in deforming the limb soft tissue by $11 \%$ are not readily solvable. To obtain an estimate of this force $(F)$, it is assumed that the whole mass effectively moves with an 
amplitude of $5.5 \%$ of the forearms length, and the motion is a simple harmonic oscillation (equation 4). Figure 4 showed that the sectors expand and contract with only a small phase shift compared to their period, giving an equivalent motion to all the soft tissue of the forearm moving one way then the other. Using

where

$$
F=-m \cdot(2 \pi f)^{2} \cdot x
$$

$m$ - mass of soft tissue, $1.14 \mathrm{~kg}$.

$f$ is the frequency of the soft tissue, from Table 1.

and $x$ is the displacement of soft tissue mass, from Table 1 .

For the whole forearm gives a force of $117 \mathrm{~N}$ associated with soft tissue motion during impact. This force will diminish the initial and peak forces measured by the force plate.

Energy transmitted by the wave was estimated to be $70 \%$ of the kinetic energy lost by the forearm at impact. Some of the energy transmitted in this form would be passively dissipated within the medium, due to complex nature of the stress strain relation in the visco-elastic media. Mechanical boundaries between tissues will also attenuate tissue wave motion due to mode conversions, reflections and refractions not being one hundred percent efficient (Graff, 1991). This would be most noticeable at joints in bent limbs and where the tissue wave propagates into an adjacent structure with different stress strain properties such as the torso. Zatsiorsky \& Prilutsky (1987) performed an analysis of soft and stiff landings from a drop where the subject used varying degrees of active muscular control. They found that up to 75 $\%$ of the mechanical energy of the body could be dissipated passively. The value for the energy carried by the tissue wave calculated here is in good agreement with their value for energy dissipation.

Previous studies have examined the marker data over the entire range of motion of the whole limb whereas the sector method used here is independent of whole limb motion and the markers were only examined after contact had occurred. As the whole limb motion is usually much greater than the motions at impact the frequencies of the whole limb motion dominate. Including both the whole limb motion and impact motion together can produce a step function in the position data. When the data is filtered ringing will occur and the high power low frequency components will smother the higher frequency components.

In conclusion, studies have shown that intra-segmental tissue motion occurs during impacts but researchers have normally only been interested in removing this skin marker artifact. The measures made in this study show that this skin movement artifact is dependent to a large extent on the underlying soft tissue motion. With a large mass of tissue involved in this relatively high frequency intra-segmental motion, significant intra-segmental force had to have been present. For the activity examined the soft tissue motion also accounted for the majority of the energy transfer and dissipation during the impact. An understanding of how much energy can be passively transferred or dissipated may prove useful in calculating potential injury to tissue. It seems that the skin marker artifact needs to be examined in its own right before it is removed from kinetic calculations during impacts.

\section{REFERENCES}

Aerts, P., Ker, R.F., Ilsley, D.W., \& Alexander, R.M. (1995). The mechanical properties of the human heel pad: A paradox resolved. Journal of Biomechanics, 28, 1299-1308.

Andriacchi, T.P., Alexander, E.J., Toney, M.K., Dyrby, C., \& Sum, J. (1997). A point cluster method for in vivo motion analysis: applied to a study of knee kinematics. Journal of Biomechanical Engineering, 120, 743-749. 
Cappozzo, A, Catani, F., Leardini, A., Benedetti, M.G., \& Della Croce, U. (1996). Position and orientation in space of bones during movement: experimental artifacts. Clinical Biomechanics, 11, 90-100.

Clarys, J.P.,Martin, A.D., \& Drinkwater, D.T. (1984). Gross tissue weights in the human body by cadaver dissection. Human Biology, 54, 459-473.

Clarys, J.P., \& Marfell-Jones, M.J. (1986). Anthropometric prediction of component tissue masses in the minor limbs of the human body. Human Biology, 58, 761-769.

Fuller, J., Liu, L.-J., Murphy, M.C., \& Mann, R.W. (1997). A comparison of lower-extremity skeletal kinematics measured using skin- and pin-mounted markers. Human Movement Science, 16, 219-242.

Fung, Y.C. (1981). Biomechanics. Mechanical Properties of Living Tissue. Springer-Verlag New York.

Graff, K.F. (1991). Wave Motion in Elastic Solids. Dover Publications Inc.

Gruber, K., Ruder, H., Denoth, J., \& Schneider, K. (1998). A comparative study of impact dynamics: wobbling mass model versus rigid body model. Journal of Biomechanics, 31, 439-444.

Karlsson, D., \& Lundberg, A. (1994). Accuracy estimation of kinematic data derived from bone anchored external markers. In Proceedings of the 3rd International Symposium on 3-D Analysis of Human Motion. Hasselbacken Conference Center Stockholm, Sweden, pp. 27-30.

Lafortune, M.A., Lambert, C., \& Lake, M. (1992). Skin marker displacement at the knee joint. In Proceedings of the Second North American Congress on Biomechanics, Chicago.

Liu, W., \& Nigg, B.M. (2000). A mechanical model to determine the influence of masses and mass distribution on the impact force during running. Journal of Biomechanics, 33, 219-224.

Maurel, W., Thalmann, D., Wu, Y., \& Magnenat-Thalmann, N. (1998). Biomechanical Models for Soft Tissue Simulation. Vol. 166. Springer-Verlag, New York.

Pain, M.T. \& Challis, J.H., (2001a) The role of the heel pad and shank soft tissue during impacts: A further resolution of a paradox. Journal of Biomechanics, 34, 327-333.

Pain, M.T.G., \& J.H. Challis, J.H. (2001b). A high resolution technique for determining body segment inertial parameters and their variation due to soft tissue motion. Journal of Applied Biomechanics, 17, 326-324.

Reinschmidt, C. (1996). Three dimensional tibiocalcaneal and tibiofemoral kinematics during human locomotion - measured with external and bone markers. Unpublished doctoral thesis, The University of Calgary, Alberta.

Reinschmidt, C., Bogert, A.J. van den, Nigg, B.M., Lundberg, A., \& Murphy, N. (1997). Effect of skin movement on the analysis of skeletal knee joint motion during running. Journal of Biomechanics, 30, 729-732.

Zatsiorsky, V.Z., \& Prilutsky, B.I. (1987). Soft and stiff landing. In: Biomechanics $X-B$. (Editor Jonsson, B.) Human Kinetics Publishers, Inc. Champaign, Illinois., pp. 739743. 